\title{
Risk factors for patellofemoral pain: a systematic review and meta-analysis
}

\author{
Bradley S Neal, ${ }^{1,2}$ Simon D Lack, ${ }^{1,2}$ Nienke E Lankhorst, ${ }^{3}$ Andrew Raye, ${ }_{1}^{1}$ \\ Dylan Morrissey, ${ }^{1,4}$ Marienke van Middelkoop ${ }^{3}$
}

${ }^{1}$ Sports and Exercise Medicine, Queen Mary University of London, London, UK

2Pure Sports Medicine, London, UK

${ }^{3}$ Department of General Practice, Erasmus MC, Rotterdam, The Netherlands ${ }^{4}$ Physiotherapy Department, Barts Health NHS Trust, London, UK

\section{Correspondence to}

Dr Marienke van Middelkoop, Department of General Practice, Erasmus MC, University Medical Center Rotterdam, Rotterdam CA 3000, The Netherlands; m.vanmiddelkoop@erasmusmc. $\mathrm{nl}$

Accepted 6 August 2018 Published Online First 21 September 2018
Check for updates

(C) Author(s) (or their employer(s)) 2019. No commercial re-use. See rights and permissions. Published by BMJ.

To cite: Neal BS, Lack SD, Lankhorst NE, et al. Br J Sports Med 2019:53:270-281.

\section{ABSTRACT}

Background Patellofemoral pain (PFP) is a prevalent condition commencing at various points throughout life. We aimed to provide an evidence synthesis concerning predictive variables for PFP, to aid development of preventative interventions.

Methods We searched Medline, Web of Science and SCOPUS until February 2017 for prospective studies investigating at least one potential risk factor for future PFP. Two independent reviewers appraised methodological quality using the Newcastle-Ottawa Scale. We conducted meta-analysis where appropriate, with standardised mean differences (SMD) and risk ratios calculated for continuous and nominal scaled data. Results This review included 18 studies involving 4818 participants, of whom 483 developed PFP (heterogeneous incidence 10\%). Three distinct subgroups (military recruits, adolescents and recreational runners) were identified. Strong to moderate evidence indicated that age, height, weight, body mass index (BMI), body fat and Q angle were not risk factors for future PFP. Moderate evidence indicated that quadriceps weakness was a risk factor for future PFP in the military, especially when normalised by BMI (SMD $-0.69, \mathrm{Cl}-1.02,-0.35)$. Moderate evidence indicated that hip weakness was not a risk factor for future PFP (multiple pooled SMDs, range -0.09 to -0.20$)$, but in adolescents, moderate evidence indicated that increased hip abduction strength was a risk factor for future PFP (SMD 0.71, CI 0.39, 1.04).

Conclusions This review identified multiple variables that did not predict future PFP, but quadriceps weakness in military recruits and higher hip strength in adolescents were risk factors for PFP. Identifying modifiable risk factors is an urgent priority to improve prevention and treatment outcomes.

\section{INTRODUCTION}

Patellofemoral pain (PFP) is characterised by diffuse retropatellar or peripatellar symptoms throughout activities that load the knee during flexion, such as running, stair descent or squatting. ${ }^{1}$ PFP is a common pathology in both adolescents ${ }^{2}$ and adults, ${ }^{3}$ with prevalence in the general population reported as $22.7 \%{ }^{4}$ However, the factors associated with PFP development and the incidence of the condition across a variety of populations remains under-evaluated due to limited prospective data and the homogeneity of studied populations. ${ }^{45}$ As PFP is reported to be common across the lifespan and may be the precursor to patellofemoral osteoarthritis, ${ }^{67}$ an improved understanding of the factors associated with the development of PFP and its incidence in differing populations is essential to prevent symptoms.
With the incidence of PFP reported to be high ${ }^{4}$ and symptoms persisting despite evidence based interventions, ${ }^{8}$ further investigation is warranted to understand variables that are associated with PFP development and subsequently deliver evidence based preventative strategies. In 1992, Van Mechelen et al presented a theoretical model described as the 'sequence of prevention' for sports injury (see figure 1) to guide injury prevention development. ${ }^{9}$ With the incidence of PFP defined across populations $^{4}$ (stage 1), an understanding of the aetiology (stage 2 ) is required to identify the variables associated with the pathology's development. A variable associated with future pathology should be manipulated as a preventative strategy within a randomised controlled trial (stage 3). The effectiveness of the implemented strategy should then be appraised by re-examining the incidence within a specific population (stage 4).

In 2012, Lankhorst et al completed a systematic review of risk factors for PFP, ${ }^{10}$ which identified a clear association between low knee extension strength and subsequent risk of PFP, irrespective of measurement method, but no associations with other investigated variables. This is likely due to the low number of included studies $(n=7)$, high data heterogeneity and data pooling being possible for just 13 out of 137 identified variables, but was unexpected given the known cross sectional association between PFP and multiple pathomechanical variables, such as muscle function and lower limb biomechanics. $^{11}$

Additional risk factors for future PFP have been reported in other systematic reviews using data from single studies. Increased navicular drop in military recruits, ${ }^{12}$ greater peak hip adduction during running ${ }^{13}$ and increased forces at foot level during both walking and running ${ }^{14}$ all increase the risk of future PFP. While these findings are statistically significant, the absence of data pooling and the small to moderate effect sizes limit their impact and clinical applicability. Given the number of subsequently published prospective studies, an updated systematic review on this topic is now appropriate.

The aim of this systematic review was to provide researchers and clinicians with an evidence synthesis concerning predictive variables for PFP, to aid the development of preventative interventions. The review was designed to synthesise the available evidence at stage 2 (aetiology) of the Van Mechelen model (see figure 1), and enable addressing stage 3 (preventative strategies). A secondary aim was to determine the incidence of PFP within the included studies, both as a 


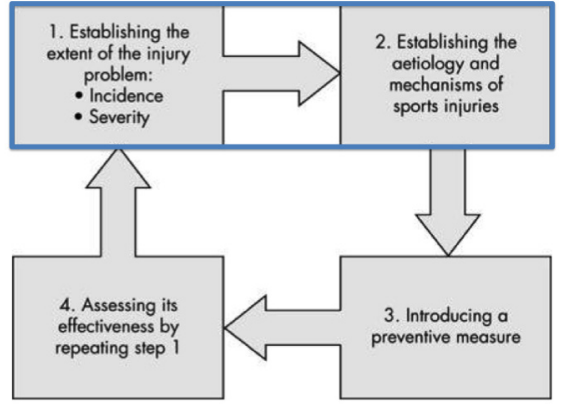

Figure 1 The Van Mechelen model of injury prediction.

heterogeneous condition and within specific homogenous cohorts. Specific objectives were to (i) establish prospective links between all investigated variables and future PFP, (ii) identify risk factors and PFP incidence specific to individual homogenous cohorts and (iii) inform future studies on PFP prevention.

\section{METHODS}

This systematic review was completed in accordance with the Preferred Reporting Items for Systematic Reviews and Meta-Analysis (PRISMA) statement ${ }^{15}$ and was registered with PROSPERO prior to completion of the initial search (registration No: CRD42016049327).

\section{Search strategy}

The search terms used by Lankhorst et $a l^{10}$ were duplicated for the purpose of this review. The following terms were used for PFP: arthralgia AND knee joint OR anterior knee pain OR (patell* OR femoropatell* OR femoro-patell* OR retropatell*) AND (pain OR syndrome OR dysfunction). Keywords used for risk factors were: risk factor OR association OR relative risk OR OR. We searched MEDLINE, Web of Science and SCOPUS from inception until February 2017, limited to papers published in the English language involving human subjects. In addition, a citing reference search was undertaken using Google Scholar up to March 2018, as well as hand searching of the reference lists of identified papers.

\section{Inclusion criteria}

A single investigator (ADR) exported all studies identified by the search strategy to Endnote X7 (Thomson Reuters, Philadelphia, USA). Eligibility criteria were adapted from the original review of Lankhorst et $a l^{10}$ : (i) studies involving male or female subjects who developed subsequent PFP (synonyms including retropatella pain, chondromalacia or anterior knee pain); (ii) at least one variable investigated as a risk factor for PFP; and (iii) prospective study designs. Studies with $<20$ PFP subjects were excluded by the review of Lankhorst et $a l,{ }^{10}$ but were included in this review. Two independent authors (BSN and NEL) reviewed all abstracts to determine eligibility. Full texts were screened where eligibility could not be determined by the abstract alone and any discrepancies were resolved at a consensus meeting.

\section{Quality assessment}

Methodological quality and risk of bias of included studies was determined by combining the Newcastle-Ottawa Scale ${ }^{16}$ (NOS) and appraising the number of events per variable. ${ }^{17}$ Eligible studies were independently rated by two authors blind to the study authors and institutions (SDL and NEL), with discrepancies resolved at a consensus meeting. The NOS contains eight categories relating to methodological quality and each study was given an eventual score out of a maximum of 8 points. A score of $0-3$ points equated to a low quality (LQ) study, a score of 4-6 points equated to a moderate quality (MQ) study, with a score of 7-8 points required for a study to be given a score of high quality (HQ). In addition, HQ or MQ studies were reduced to either MQ or LQ respectively, if they were determined to have a high risk of bias as a result of having $<10$ PFP participants for each investigated variable within their total sample. ${ }^{17}$ Inter-rater reliability of the NOS was calculated using the percentage agreement method.

\section{Data extraction}

Data related to study characteristics were initially extracted from all included studies by one author (ADR) and subsequently reviewed by a second author (BSN). This included participant numbers (separating those who developed PFP and those who did not), characteristics of these groups (such as population), study duration and publication details (author and year). A second author (BSN) extracted all data pertaining to potential risk variables to be included in the meta-analysis. Means and SD were extracted for variables of interest, which included (but were not limited to) anthropometrics and demographics (such as sex, body mass index (BMI)), biomechanical variables (such as kinematics and kinetics) and muscle function (such as strength or onset timing).

\section{Statistical methods}

Statistical analyses were undertaken using Review Manager 5.0 (The Cochrane Collaboration, Copenhagen, Denmark). Analyses were completed initially by one author (BSN) and subsequently reviewed by a second author (SDL). Means and SDs were extracted for continuous scaled variables and used to calculate a standardised mean difference (SMD) with $95 \%$ confidence intervals (CIs). Calculated individual or pooled SMDs were categorised as small (0.59), medium (0.60-1.19) or large (1.20). ${ }^{18}$ For nominal scaled variables, raw counts of injured and uninjured participants (eg, PFP incidence in men and women) were extracted and used to calculate risk ratios (RR) with 95\% CIs, with a small effect indicated by an $R R \geq 2.0$ and a large effect by an $R R \geq 4.0 .^{18}$

Data were pooled and have been presented as both a heterogeneous PFP cohort and further pooled by specific homogeneous subgroup where possible. Where methodological approaches between studies were deemed to be adequately comparable, a meta-analysis was performed and the level of statistical heterogeneity for pooled data determined using $I^{2}$ statistics (heterogeneity defined as $\left.I^{2}>50 \%, \mathrm{P}<0.05\right)$. Random effects were used due to the variation in study methods and populations, and the typically low number of studies, therefore reducing the possibility of a type 1 error. ${ }^{19}$

Only outcomes incorporating data from a minimum of two studies are presented in the main body of the review, due to the risk of reporting inappropriate levels of evidence where data pooling was not possible.

\section{Evidence based recommendations}

Levels of evidence were assigned to each calculated variable (pooled or otherwise), as described by Van Tulder et al, ${ }^{20}$ which 


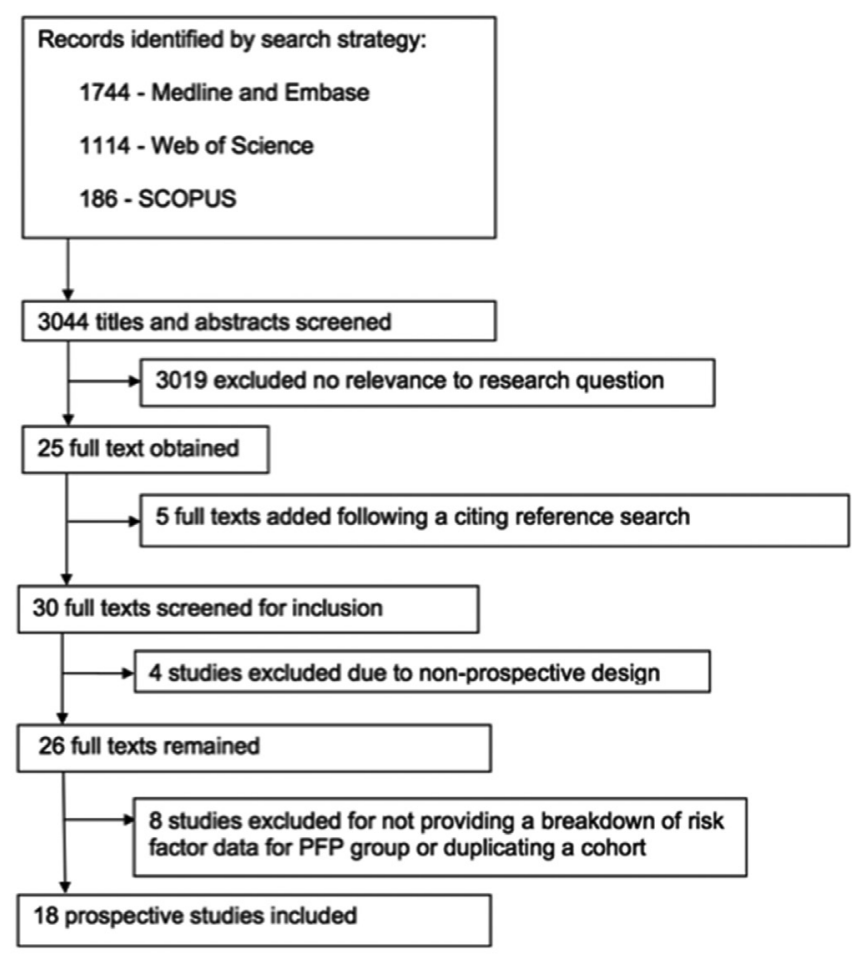

Figure 2 PRISMA search flowchart.

incorporate both assigned methodological quality of included studies and statistical outcomes.

\section{Strong evidence}

Pooled results derived from three or more studies, including a minimum of two high quality studies that are statistically homogenous.
Moderate evidence

Pooled results derived from multiple studies, including at least one high quality study, that are statistically heterogeneous; or from multiple moderate or low quality studies which are statistically homogenous.

Limited evidence

Results from one high quality study or multiple moderate or low quality studies that are statistically heterogeneous.

Very limited evidence

Results from one moderate or low quality study.

\section{RESULTS}

Search results

The search resulted in 3044 titles and abstracts being identified for screening. Following the removal of duplicates and studies that did not meet the inclusion criteria of the review, 18 studies involving a total of 4818 participants were included (see figure 2), ${ }^{21-38} 483$ of whom went on to develop symptoms consistent with PFP. This is indicative of a heterogeneous PFP incidence of $10 \%$. Extracted data relating to study characteristics are presented in table 1.

\section{Subgroups and PFP incidence}

Three distinct subgroups were identified during the data extraction process. There were a total of seven studies involving military recruits, ${ }^{21}$ 23-25 283637 six studies involving adolescents $^{22} 2627293138$ and five studies involving recreational runners. ${ }^{30}$ 32-35 Studies involving military recruits involved a total of 2435 participants, 280 of whom went on to develop PFP, reflective of an incidence of 11\% (range 3-43\%). Studies involving adolescents involved a total of 1118 participants, 128 of whom went on to develop PFP, reflective of an incidence of $11 \%$ (range $5-15 \%$ ). Studies involving recreational runners

Table 1 Study characteristics

\begin{tabular}{|c|c|c|c|c|c|c|c|}
\hline Study & NOS score & Risk of bias & Cohort & PFP & Sample size & Incidence (\%) & $\begin{array}{l}\text { Study duration } \\
\text { (months) }\end{array}$ \\
\hline Boling et $a l^{21}$ & $\mathrm{H}$ & L & Military (USA) & $40(\mathrm{M}=16, \mathrm{~F}=24)$ & 1319 & 3 & 30 \\
\hline Duvigneaud et $\left.a\right|^{25}$ & $\mathrm{H}$ & L & Military (Belgium) & $26(F=26)$ & 62 & 42 & 1.5 \\
\hline Finnoff et $a l^{26}$ & MD & $\mathrm{H}$ & Adolescents (USA) & $5(M=2, F=3)$ & 98 & 5 & 9 \\
\hline Foss et $a l^{22}$ & $\mathrm{H}$ & $\mathrm{L}$ & Adolescents (USA) & $39(F=39)$ & 262 & 15 & 24 \\
\hline Herbst et $a l^{27}$ & $\mathrm{H}$ & L & Adolescents (USA) & $38(F=38)$ & 255 & 15 & 12 \\
\hline Hetsroni et $a l^{28}$ & $\mathrm{H}$ & $\mathrm{L}$ & Military (Israel) & $61(\mathrm{M} / \mathrm{F}=?)$ & 405 & 15 & 4 \\
\hline Holden et $a l^{29}$ & MD & $\mathrm{H}$ & Adolescents (Ireland) & $8(F=8)$ & 76 & 11 & 24 \\
\hline Luedke et $a l^{30}$ & MD & $\mathrm{H}$ & Recreational runners (USA) & $3(\mathrm{M}=1, \mathrm{~F}=2)$ & 57 & 5 & 12 \\
\hline Milgrom et $a^{23}$ & MD & $\mathrm{L}$ & Military (Israel) & $60(M=60)$ & 390 & 15 & 3.5 \\
\hline Myer et $a l^{31}$ & MD & $\mathrm{H}$ & Adolescents (USA) & 14 & 145 & 10 & 9 \\
\hline Noehren ${ }^{32}$ & MD & $\mathrm{H}$ & Recreational runners (USA) & $15(F=15)$ & 400 & 3 & 24 \\
\hline Ramskov et $a \beta^{33}$ & $\mathrm{H}$ & L & $\begin{array}{l}\text { Recreational } \\
\text { runners (Denmark) }\end{array}$ & $24(\mathrm{M}=10, \mathrm{~F}=14)$ & 629 & 4 & 12 \\
\hline Thijs et $a l^{36}$ & $\mathrm{H}$ & $\mathrm{L}$ & Military (Belgium) & $36(M=25, F=11)$ & 84 & 43 & 1.5 \\
\hline Thijs et $a l^{34}$ & MD & $\mathrm{H}$ & $\begin{array}{l}\text { Recreational } \\
\text { runners (Belgium) }\end{array}$ & $17(\mathrm{M}=1, \mathrm{~F}=16)$ & 102 & 17 & 2.5 \\
\hline Thijs et $a l^{35}$ & MD & $\mathrm{H}$ & $\begin{array}{l}\text { Recreational } \\
\text { runners (Belgium) }\end{array}$ & $16(F=16)$ & 77 & 21 & 2.5 \\
\hline Van Tiggelen et $a P^{37}$ & $\mathrm{H}$ & L & Military (Belgium) & $31(\mathrm{M}=31)$ & 96 & 32 & 1.5 \\
\hline Van Tiggelen et $a^{24}$ & $\mathrm{H}$ & $\mathrm{L}$ & Military (Belgium) & $26(M=26)$ & 79 & 33 & 1.5 \\
\hline Witvrouw et $a \beta^{38}$ & MD & $\mathrm{H}$ & Adolescents (Belgium) & $24(\mathrm{M}=11, \mathrm{~F}=13)$ & 282 & 9 & 24 \\
\hline
\end{tabular}

F, female; H, high; L, low; M, male; MD, moderate; NOS, Newcastle-Ottawa Scale; PFP, patellofemoral pain. 
involved a total of 1265 participants, 75 of whom went on to develop PFP, reflective of an incidence of 6\% (range 4-21\%).

\section{Quality assessment}

After evaluation of study quality and the risk of bias, ${ }^{16} 17$ a total of nine HQ studies 212224252728333637 and a further nine MQ studies were identified. ${ }^{23} 26$ 29-32 343538 Mean percentage agreement for the NOS was 95\% (range 89-100\%), indicating high inter-rater reliability (see table 2 ). The questions with the lowest percentage agreement were question No 5 (does the study control for any confounding variables) and question No 7 (was follow-up time clearly defined).

\section{Anthropometrics and demographics}

Data pooling was possible for seven individual variables (sex, height, weight, BMI, body fat percentage, age and limb length).

\section{Sex}

There is moderate evidence from three $\mathrm{HQ}^{2133} 36$ and four $\mathrm{MQ}^{26} 303438$ studies that sex is not a risk factor for future PFP $\left(I^{2}=73 \%\right.$, RR 1.33 , CI $\left.0.76,2.34\right)$ (see figure 3 ). This outcome does not change when pooling data only for military subjects (moderate evidence, $I^{2}=91 \%$, RR 0.82, CI 0.25, 2.74), adolescents (moderate evidence, $I^{2}=0 \%$, RR 1.23 , CI $0.38,2.07$ ) or recreational runners (moderate evidence, $I^{2}=76 \%$, RR 3.08, CI $0.59,15.99)$. While subgroup data pooling was non-significant, six of the seven included studies that reported data on sex had a greater proportion of women in their PFP cohort, ${ }^{21} 2630333638$ the highest of which was observed in the recreational runner subgroup.

\section{Height}

There is strong evidence from five $\mathrm{HQ}^{24} 25333637$ and seven $\mathrm{MQ}^{23} 262931343538$ studies that height is not a risk factor for future PFP $\left(I^{2}=0 \%, \mathrm{SMD}-0.08, \mathrm{CI}-0.21,0.05\right)$ (see figure 4). This outcome does not change when pooling data for only military recruits (strong evidence, $I^{2}=41 \%$, SMD -0.15 , CI $-0.42,0.12$ ), adolescents (moderate evidence, $I^{2}=0 \%$, SMD 0.06 , CI $-0.23,0.35$ ) or recreational runners (moderate evidence, $I^{2}=0 \%$, SMD $\left.-0.15, \mathrm{CI}-0.43,0.13\right)$.

\section{Weight}

There is strong evidence from five $\mathrm{HQ}^{24} 25333637$ and seven

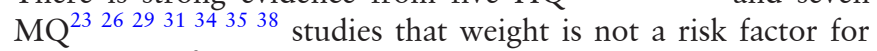
future PFP $\left(I^{2}=0 \%\right.$, SMD 0.02, CI $\left.-0.11,0.16\right)$ (see figure 5). This outcome does not change when pooling data for only military recruits (strong evidence, $I^{2}=0 \%$, SMD 0.05 , CI $-0.12,0.23$ ), adolescents (moderate evidence, $I^{2}=28 \%$, SMD -0.10 , CI $-0.46,0.25$ ) or recreational runners (moderate evidence, $I^{2}=0 \%$, SMD $\left.0.10, \mathrm{CI}-0.18,0.37\right)$.

\section{Body mass index}

There is strong evidence from four $\mathrm{HQ}^{22} 253337$ and three $\mathrm{MQ}^{26} 3435$ studies that BMI is not a risk factor for future PFP $\left(I^{2}=33 \%\right.$, SMD 0.10 , CI -0.12 to 0.32 ) (see figure 6 ). This outcome does not change when pooling data for only military recruits (moderate evidence, $I^{2}=65 \%$, SMD 0.09 , CI -0.48 to 0.65 ), adolescents (moderate evidence, $I^{2}=75 \%$, SMD 0.23 , CI -0.72 to 1.18 ) or recreational runners (moderate evidence, $I^{2}=0 \%$, SMD $0.15, \mathrm{CI}-0.13$ to 0.43 ).
Body fat percentage

There is moderate evidence from one HQ study ${ }^{22}$ and one MQ study $^{38}$ that body fat percentage is not a risk factor for future PFP in adolescents $\left(I^{2}=0 \%, \mathrm{SMD}-0.13\right.$, CI $\left.-0.40,0.13\right)$ (see figure 7). This variable was not investigated in either military recruits or recreational runners.

\section{Age}

There is strong evidence from three $\mathrm{HQ}^{24} 3336$ and five MQ studies 2931323435 that age is not a risk factor for future PFP $\left(I^{2}=13 \%\right.$, SMD 0.06 , CI $-0.13,0.25$ ) (see figure 8 ). This outcome does not change when pooling data for only military recruits (moderate evidence, $I^{2}=0 \%$, SMD -0.05 , CI $-0.36,0.27$ ), adolescents (limited evidence, $I^{2}=80 \%$, SMD 0.04 , CI $-0.98,1.07$ ) or recreational runners (moderate evidence, $I^{2}=0 \%$, SMD 0.16, CI $\left.-0.09,0.40\right)$.

\section{Limb length}

There is limited evidence from two MQ studies ${ }^{23} 26$ that limb length is not a risk factor for future PFP $\left(I^{2}=0 \%\right.$, SMD -0.01 , CI $-0.28,0.25)$. This variable was not investigated in recreational runners and no data pooling was possible within any individual subgroups.

\section{Lower limb alignment}

Data pooling was only possible for static $Q$ angle. Limited evidence from one $\mathrm{HQ}^{21}$ and one $\mathrm{MQ}$ study ${ }^{35}$ indicates that $\mathrm{Q}$ angle is not a risk factor for future PFP $\left(I^{2}=0 \%\right.$, SMD 0.06 , CI $-0.22,0.33$ ) (see figure 9). No data pooling was possible for any identified subgroup.

\section{Strength measures}

\section{Quadriceps strength}

When pooling all available data for quadriceps strength, regardless of cohort or measurement method, there is strong evidence that quadriceps weakness is a risk factor for future PFP (moderate evidence, $I^{2}=65 \%$, small SMD -0.32 , CI -0.42 to -0.22 ).

Data pooling was only possible for the military subgroup for all quadriceps strength measures. There is moderate evidence from two HQ studies ${ }^{25} 37$ that quadriceps weakness is a risk factor for future PFP when measured with an isokinetic dynamometer concentrically at $60 \% \quad\left(I^{2}=0 \%\right.$, moderate SMD -0.66 , CI $-0.99,-0.32$ ) (see figure $10 \mathrm{~A}$ ) or concentrically at $240 \%$ s $\left(I^{2}=17 \%\right.$, small SMD -0.49 , CI -0.85 to -0.12 ) (see figure 10B).

For normalised quadriceps strength measured with an isokinetic dynamometer, there is moderate evidence from two HQ studies ${ }^{25} 37$ that quadriceps weakness is a risk factor for future PFP when normalised by body mass at $60 \%\left(I^{2}=0 \%\right.$, moderate SMD -0.61 , CI -0.94 to -0.28 ) (see figure $10 \mathrm{C}$ ) or at $240 \% \mathrm{~s}\left(I^{2}=0 \%\right.$, small SMD -0.53 , CI -0.87 to -0.20$)$ (see figure 10D). When normalised by BMI, moderate evidence remains that quadriceps weakness is a risk factor for future PFP when measured at both $60 \% \mathrm{~s}\left(I^{2}=0 \%\right.$, moderate SMD -0.69 , CI -1.02 to-0.35) (see figure $10 \mathrm{E}$ ) and $240 \%\left(I^{2}=0 \%\right.$, small SMD -0.51 , CI -0.84 to -0.18 ) (see figure $10 \mathrm{~F}$ ).

For quadriceps strength measured isometrically with a handheld dynamometer (HHD), there is moderate evidence from one $\mathrm{HQ}^{21}$ and one MQ study ${ }^{23}$ that quadriceps strength is not a risk factor for future PFP $\left(I^{2}=82 \%\right.$, small SMD -0.25 , CI $-0.74,0.25)$. 


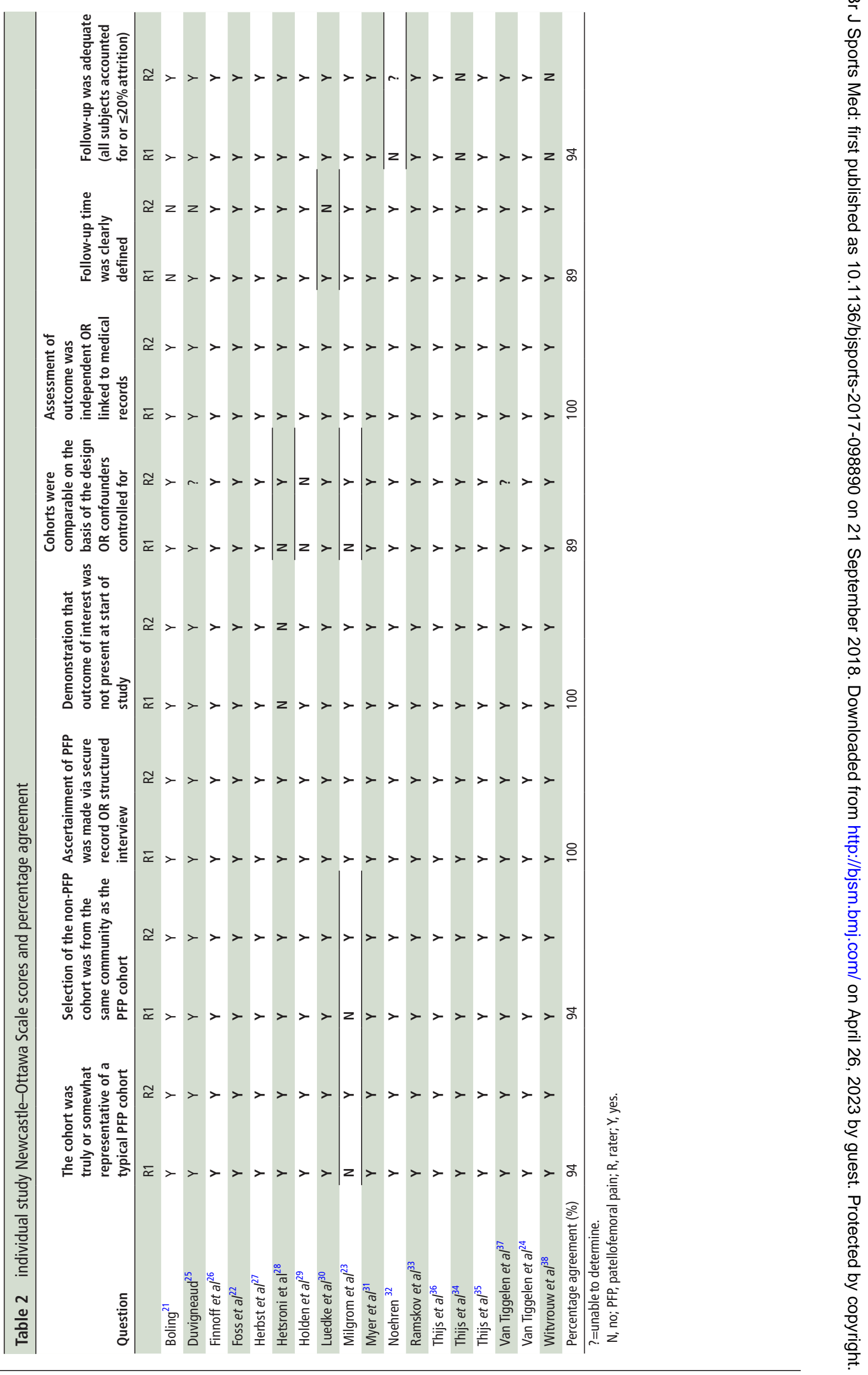




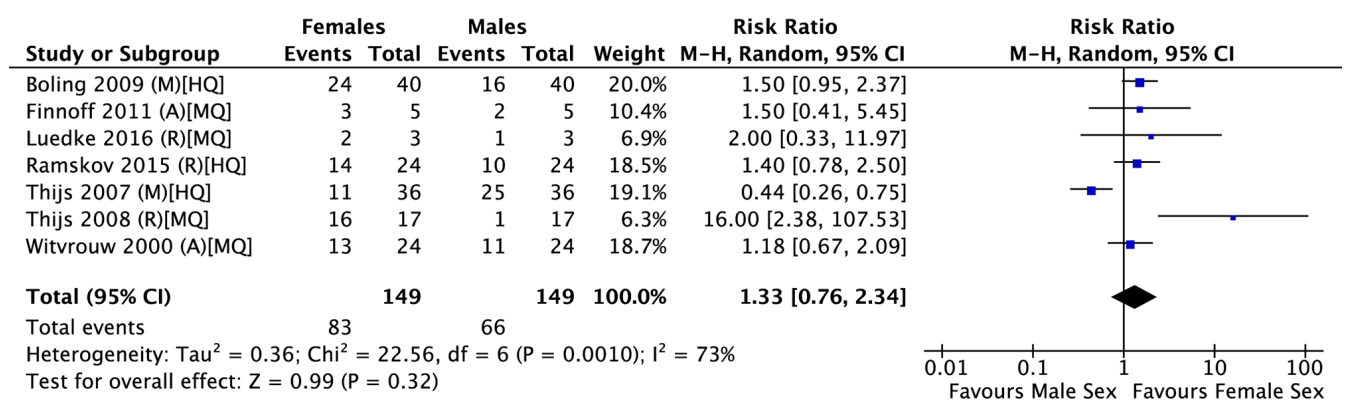

Figure 3 Sex. Forrest plot detailing risk ratios for sex when comparing participants who developed patellofemoral pain (PFP) with controls. A, adolescents; $H Q$, high quality; $M$, military recruits; $M Q$, medium quality; $R$, recreational runners.

\section{Hamstrings strength}

There is moderate evidence from two HQ studies 2537 that hamstring strength is not a risk factor for future PFP in the military when measured with an isokinetic dynamometer concentrically at $60 \% \mathrm{~s}\left(I^{2}=0 \%, \mathrm{SMD}-0.09\right.$, CI $\left.-0.42,0.24\right)$ or $240^{\circ} / \mathrm{s}$ $\left(I^{2}=0 \%, \mathrm{SMD}-0.10, \mathrm{CI}-0.43,0.22\right)$. This variable was not investigated in either adolescents or recreational runners.

\section{Hip strength}

There is moderate evidence from one $\mathrm{HQ}^{21}$ and two $\mathrm{MQ}$ studies $^{26} 35$ that hip extension $\left(I^{2}=0 \%\right.$, SMD -0.18 , CI $-0.44,0.09$ ) (see figure $11 \mathrm{~A}$ ), hip internal rotation $\left(I^{2}=20 \%\right.$, SMD -0.09 , CI $-0.42,0.23$ ) (see figure 11B) and hip external rotation strength $\left(I^{2}=0 \%, \mathrm{SMD}-0.17, \mathrm{CI}-0.43,0.10\right)$ (see figure $11 \mathrm{C}$ ), measured isometrically with a HHD, are not risk factors for future PFP. There is also limited evidence from two MQ studies ${ }^{26} 35$ that both hip adduction strength $\left(I^{2}=0 \%\right.$, SMD $-0.20, \mathrm{CI}-0.67,0.28$ ) (see figure 11D) and hip flexion strength $\left(I^{2}=52 \%\right.$, SMD $\left.-0.08, \mathrm{CI}-0.82,0.67\right)$ (see figure 11E) are not risk factors for future PFP when measured with a HHD. No data pooling was possible for any identified subgroup for these strength measures.

There is moderate evidence from two $\mathrm{HQ}^{2127}$ and two $\mathrm{MQ}^{2635}$ that hip abduction strength is not a risk factor for future PFP $\left(I^{2}=86 \%\right.$, SMD 0.25 , CI $\left.-0.38,0.88\right)$ (see figure $12 \mathrm{~A}$ ) when measured isometrically with a HHD. When the data were pooled for the adolescent cohort, there is moderate evidence from one $\mathrm{HQ}^{27}$ and one MQ study ${ }^{26}$ that increased hip abduction strength is a risk factor for future PFP $\left(I^{2}=0 \%\right.$, SMD 0.71 , CI $\left.0.39,1.04\right)$ (see figure 12B) when measured isometrically with a HHD. Data pooling was not possible for the military or recreational runner subgroups.

\section{Biomechanics}

Dynamic knee valgus angle

Moderate evidence from one HQ study ${ }^{21}$ and one MQ study ${ }^{29}$ indicates that knee valgus angle during a jump land task is not a risk factor for future PFP $\left(I^{2}=99 \%\right.$, SMD 4.17, CI -4.19, 12.53). No data pooling was possible for any identified subgroup.

\section{Foot kinetics}

One HQ study ${ }^{36}$ and one MQ study ${ }^{34}$ investigated foot kinetics during walking and running, respectively. When these data were pooled, moderate evidence indicates no significant associations between time to peak force at any investigated region of the foot, which included the hallux, the metatarsal heads and the medial/lateral heel.

\section{DISCUSSION}

This systematic review aimed to provide a synthesis of the evidence concerning predictive variables for PFP development. Despite the inclusion of 11 additional prospective studies and 55 additional variables when compared with the previous review of Lankhorst et al, ${ }^{10}$ high data heterogeneity and a limited ability to pool data remained. Just two predictive variables, lower isokinetic quadriceps strength in the military and higher isometric hip abduction strength in adolescents, were identified. Heterogeneous incidence of PFP was 10\%, with incidence also identified

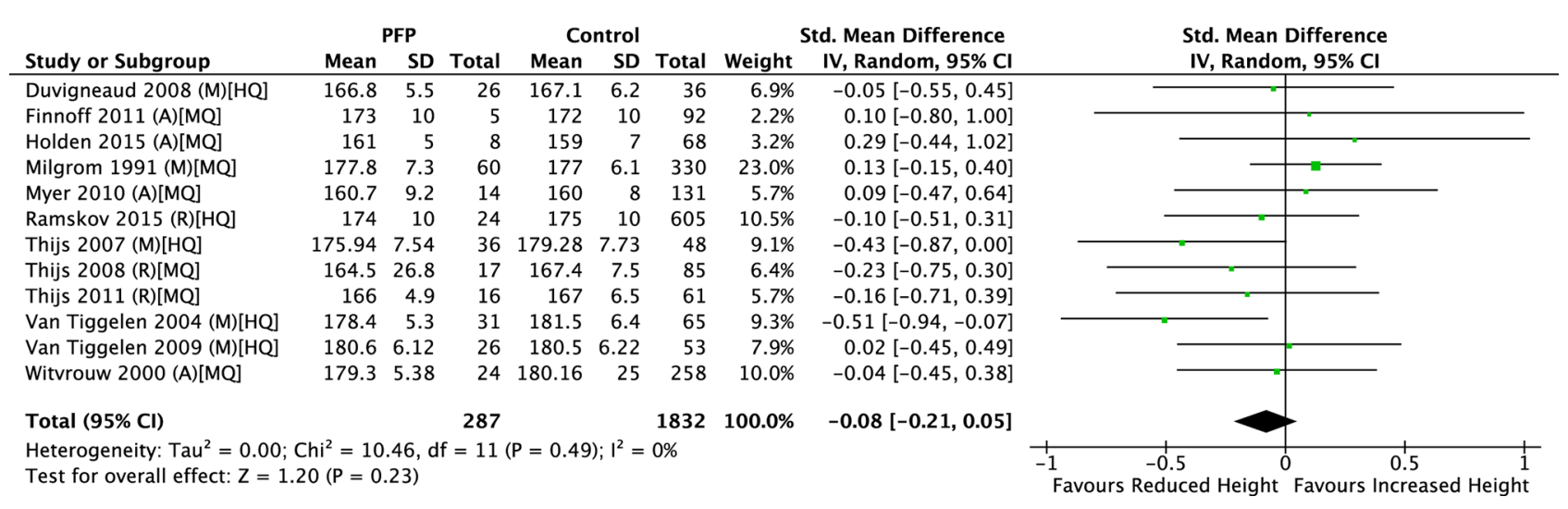

Figure 4 Height. Forrest plot detailing standardised mean differences for height when comparing participants who developed patellofemoral pain (PFP) with controls. A, adolescents; HQ, high quality; IV, inverse variance; M, military recruits; MQ, medium quality; R, recreational runners. 


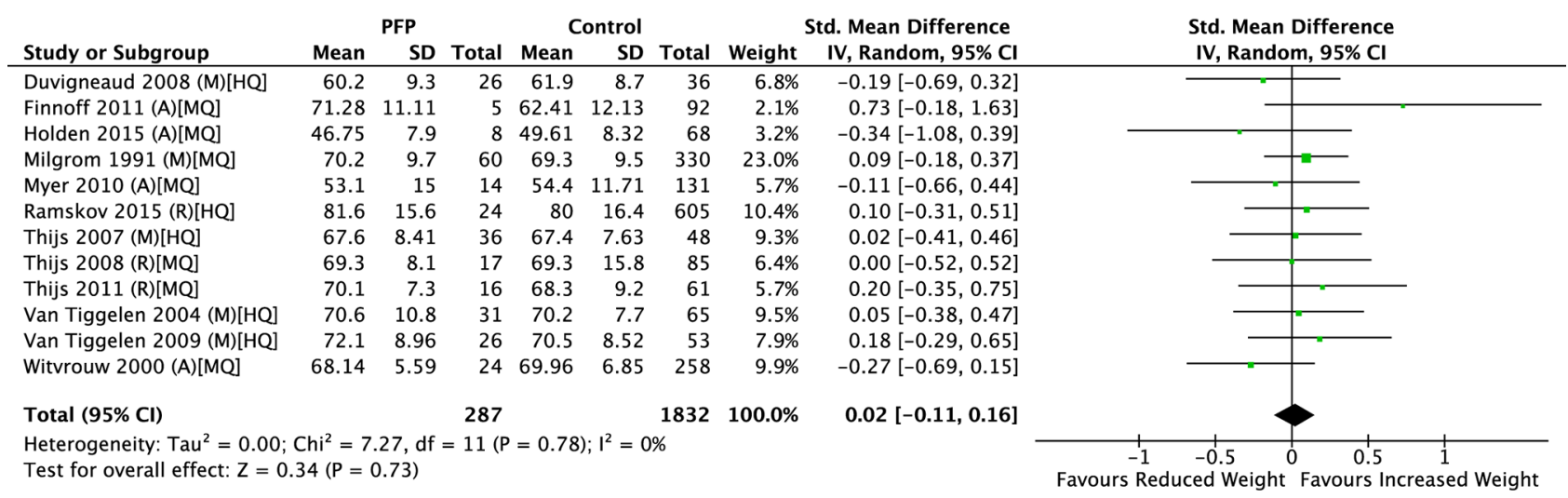

Figure 5 Weight. Forrest plot detailing standardised mean differences for weight when comparing participants who developed patellofemoral pain (PFP) with controls. A, adolescents; HQ, high quality; IV, inverse variance; M, military recruits; MQ, medium quality; R, recreational runners.

within the specific homogenous cohorts of military recruits (11\%), adolescents (11\%) and recreational runners (6\%).

Isokinetic quadriceps weakness is predictive of future PFP in a military cohort ${ }^{2537}$ and this is in agreement with the previous review of Lankhorst et al. ${ }^{10}$ Unfortunately, the strength of this evidence has not changed, as no new prospective studies using an isokinetic dynamometer to measure quadriceps strength have been published since 2011. New data from two studies investigating isometric quadriceps strength in military cohorts, ${ }^{21} 23$ not included by Lankhorst et al, demonstrated no significant association with future PFP. While this could be interpreted as conflicting evidence, it could be that isometric muscle testing may not be sensitive enough to identify military recruits at risk of PFP. This limits the clinical applicability of these results, as isometric testing with a HHD is a more accessible tool for clinicians to use when measuring muscle strength.

Quadriceps weakness was not identified as a risk factor for future PFP in an adolescent group. ${ }^{27}$ While this further validates the importance of investigating risk factors within homogenous groups, it is also important to consider the implications of these findings in relation to risk modification interventions within differing populations. A similar disparity between adult and adolescent populations has been observed cross sectionally, with no differences in hip or knee strength between adolescents with PFP and a group of asymptomatic controls matched for both age and sex, ${ }^{39}$ but significant strength deficits in adults with PFP compared with control groups. ${ }^{40}$ These findings offer indications as to why rehabilitation programmes have been shown to be of significant benefit in adults with $\mathrm{PFP}^{42}{ }^{43}$ but are of only limited additional benefit to education alone in adolescents. ${ }^{44}$

In contrast with the quadriceps data, hip muscle strength, regardless of test direction, was not a risk factor for future PFP in military recruits or recreational runners. However, increased baseline isometric hip abduction strength predicts future PFP in adolescents. ${ }^{26}{ }^{27}$ Herbst et $a l^{27}$ make the suggestion that increased hip abduction strength could be the result of greater eccentric hip abductor demands due to higher peak hip adduction during dynamic tasks. When pooling data from both military and adolescent cohorts, dynamic knee valgus angle was also not a risk factor for future PFP. However, Holden et $a l^{29}$ reported higher knee valgus displacement in adolescent women who develop PFP (mean difference $7.79^{\circ}$ ). Despite these reported kinematic deficits, hip strength and altered kinematics during dynamic tasks are consistently negatively correlated, ${ }^{45}$ contradicting this hypothesis.

A more plausible explanation for the association between increased isometric hip abduction strength and future PFP in young adolescents is a high level of physical activity, common within this age group. ${ }^{46}$ The mean age of the Herbst et $a l^{27}$ cohort is 12.7 years and may therefore have increased lower limb muscle strength as a consequence of high physical activity levels. It may be that lower limb muscle strength correlates with duration of symptoms in adolescents, with strength deficits presenting later in life when symptoms persist and if activity levels subsequently reduce. ${ }^{39}$ As a result, it is sensible to question the role of increasing muscle strength in adolescent cohorts

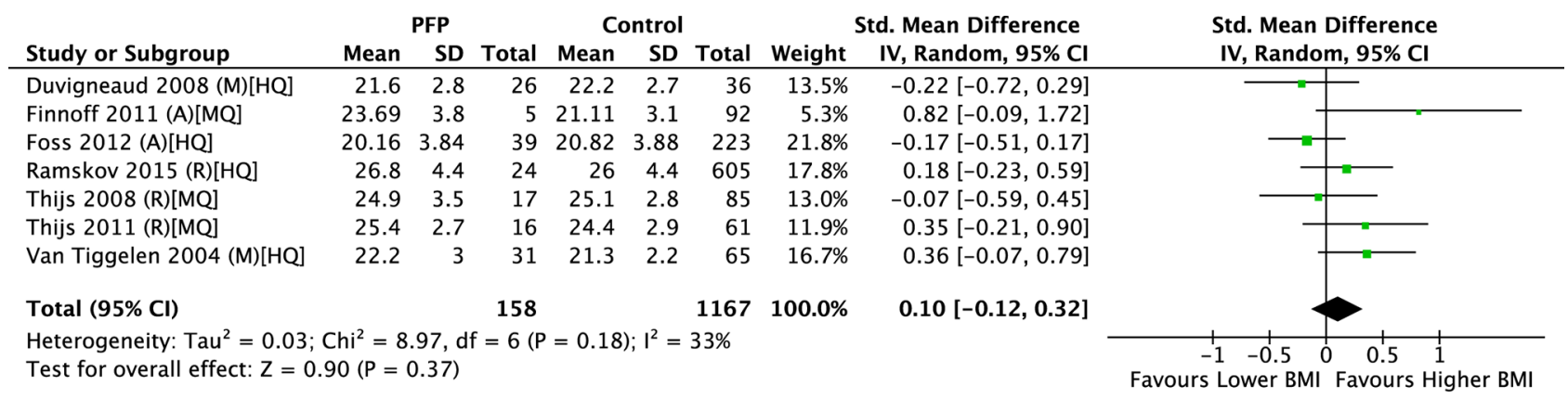

Figure 6 Body mass index (BMI). Forrest plot detailing standardised mean differences for BMI when comparing participants who developed patellofemoral pain (PFP) with controls. A, adolescents; HQ, high quality; IV, inverse variance; $M$, military recruits; MQ, medium quality; R, recreational runners. 


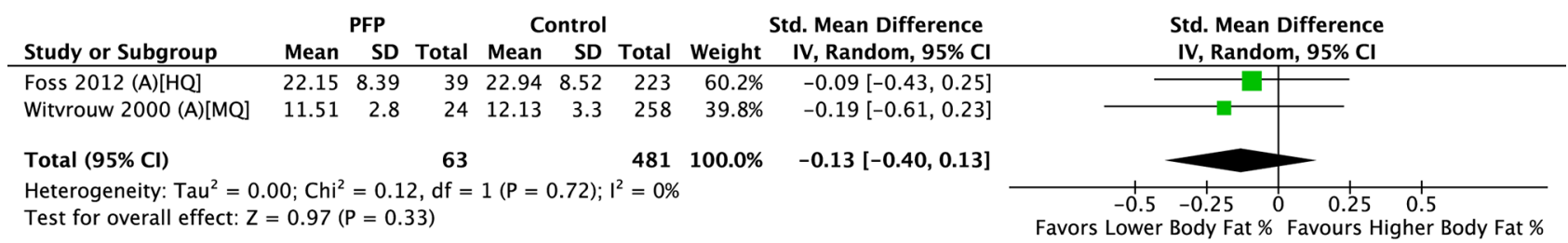

Figure 7 Body fat percentage. Forrest plot detailing standardised mean differences for body fat percentage when comparing adolescents who developed patellofemoral pain (PFP) with controls. A, adolescents; HQ, high quality; IV, inverse variance; MQ, medium quality.

as a preventative measure. It is advised that future prospective studies both report and stratify for activity levels when investigating the association between strength variables and future PFP.

In 2011, Coppack et $a l^{47}$ reported a significant reduction in PFP risk after completion of a quadriceps and gluteal strengthening programme when compared with a non-specific control group. It is surprising that an exercise intervention designed to increase quadriceps strength has not been investigated further, given both level $1^{10}$ and level $2^{47}$ evidence identifying quadriceps weakness as a preventative treatment target. While increased baseline hip abduction strength increases the risk for future PFP in adolescents, this is potentially a surrogate indicator of activity level. Given the positive results of education and load management interventions in this population, ${ }^{44}$ we suggest prioritising these interventions in future in order to reduce the incidence of PFP in adolescents.

Multiple variables often described as risk factors for future PFP, perhaps due to strong associations in cross sectional studies, were not found to be so in this meta-analysis. Participant height, weight, BMI, body fat percentage, age and Q angle did not predict future PFP in any cohort. The recent systematic review of Hart $e t a l^{48}$ reports a cross sectional association between high BMI and both PFP and patellofemoral osteoarthritis in adults, again perhaps due to a reduction in activity levels after symptom development. ${ }^{49}$ Higher BMI was not a risk factor for future PFP in either adults or adolescents, nor was a high BMI linked to intervention outcomes in participants with $\mathrm{PFP}^{48}$ While these data question the biologically plausible suggestion that a high BMI contributes to PFP development, it remains plausible that high BMI may influence treatment outcomes and this suggestion requires further investigation. ${ }^{48}$

Using data from the work of Boling et al, ${ }^{21}$ the previous 2012 review of Lankhorst $e t a l^{10}$ reported that women are at a higher risk of developing PFP within the military (OR 2.23, 95\% CI $1.16,4.10)$. Our results are in conflict with this, with pooled data from seven studies 21263033343638 identifying no significant links between female sex and PFP development. Pooling data for the identified individual subgroups is also non-significant, but a greater proportion of women developing subsequent PFP was reported in six of the seven studies. The largest proportion of women occurs among the recreational runner subgroup and this is in fact statistically significant when a fixed effects model is used for the meta-analysis, meaning observed results are most likely a result of selection bias in source studies. However, given the low number of studies $(n=3)$ and high heterogeneity, a fixed effects model is inappropriate and increases the chance of sustaining a type 1 error. ${ }^{19}$ Given the absence of a causal association between female sex and future PFP, the frequent bias towards women in trial sampling and the need to control for sex as a confounder may not be necessary.

The heterogeneous incidence of PFP was $10 \%$ in this review, demonstrating that PFP affects up to 1 in 10 persons across multiple populations. The recent systematic review of PFP incidence and prevalence by Smith et $a l^{4}$ identified a wide range of PFP incidence among military recruits (9.7-571.4 cases per 1000 person-years), which is similar to the incidence range identified by this review $(3-43 \%)$. The variance is likely explained by the four studies 24252837 included in this review not included by Smith et $a l,{ }^{4}$ and the three studies included by Smith et $a l^{4}$ not eligible for inclusion within this review. ${ }^{475051}$ The incidence range for PFP within adolescent cohorts are identical (5-15\%), despite two studies from this review ${ }^{22}$ not being included by Smith et al. ${ }^{4}$

\section{Limitations and future research directions}

This review is not without limitations, which must be considered when interpreting the results. There is currently no accepted method for determining study methodological quality or ascertaining risk of bias. While the NOS is advocated by the Cochrane Group, it is possible that using a different quality appraisal tool may have yielded different levels of eventual evidence. It should also be considered that the NOS does not have a component pertaining to the

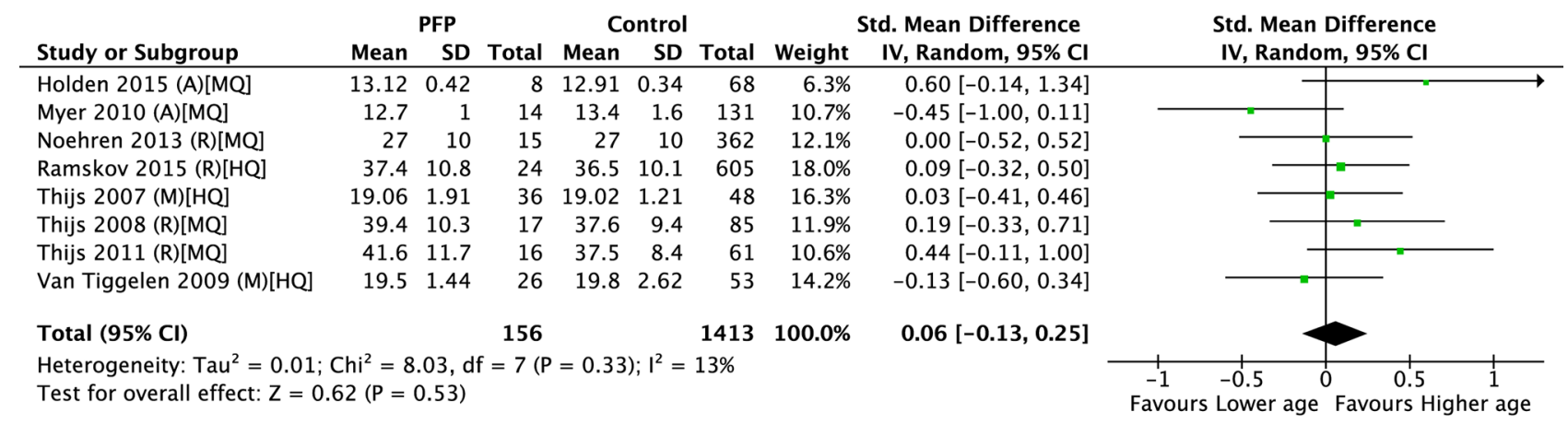

Figure 8 Age. Forrest plot detailing standardised mean differences for age when comparing participants who developed patellofemoral pain (PFP) with controls. A, adolescents; HQ, high quality; IV, inverse variance; M, military recruits; MQ, medium quality; R, recreational runners. 


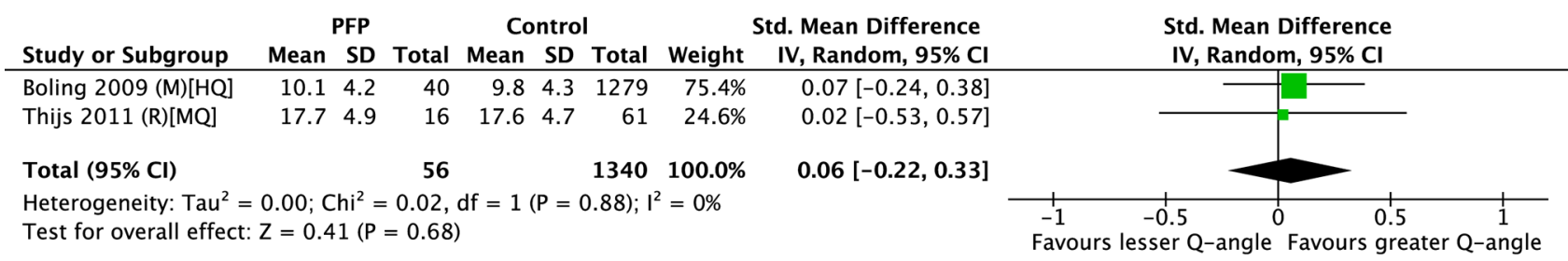

Figure 9 Q angle. Forrest plot detailing standardised mean differences for Q angle when comparing participants who developed patellofemoral pain (PFP) with controls. HQ, high quality; IV, inverse variance; M, military recruits; MQ, medium quality; R, recreational runners.

reliability of exposure data collection, focusing more on the validity of outcome data. As per the PRISMA guidelines, ${ }^{15}$ three databases were searched, but is it also possible that increasing the number of databases searched may have yielded additional studies for inclusion. An attempt was made to mitigate this risk by completing a citing reference search in Google Scholar in addition to hand searching the reference lists of included studies. It must be stressed that

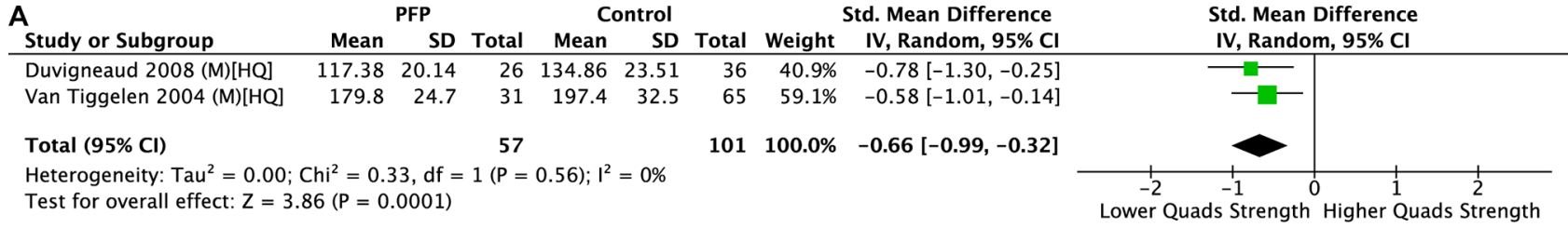

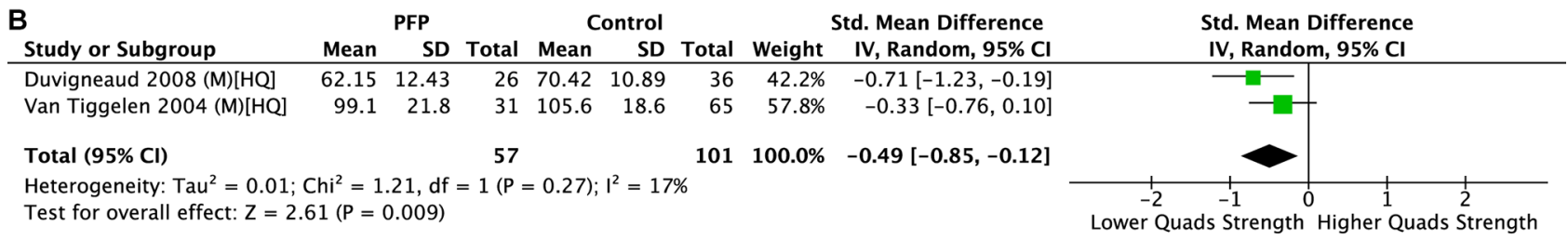

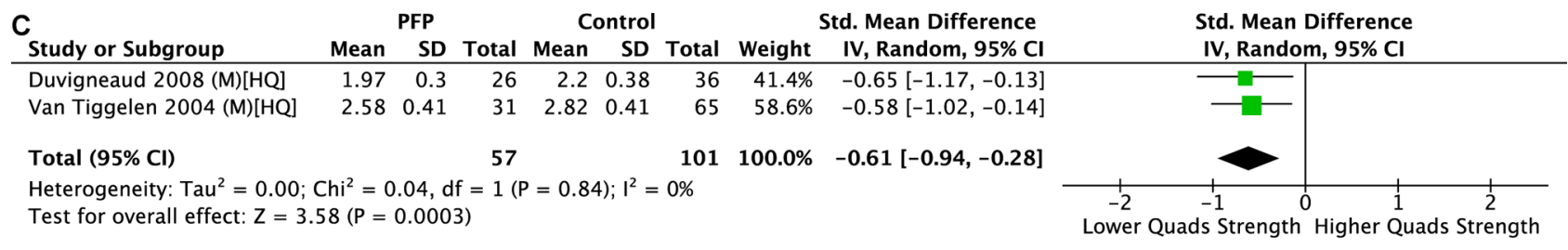

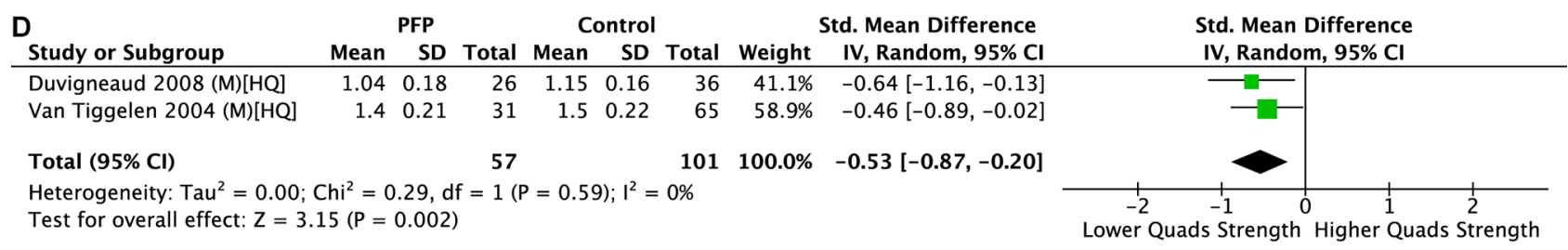

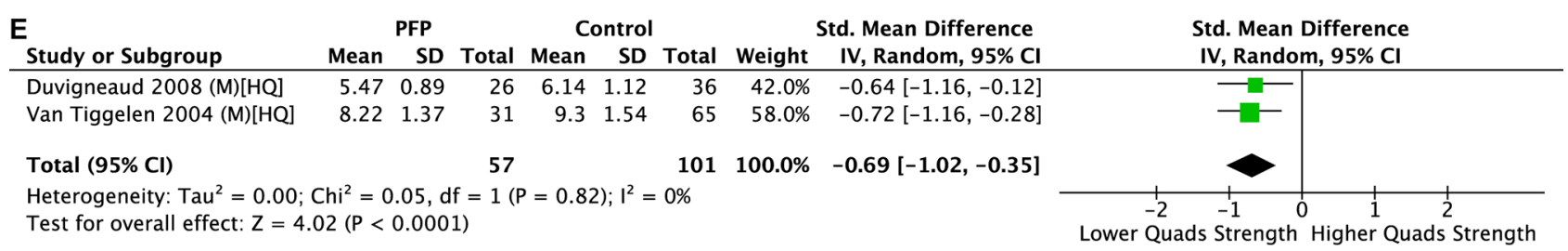

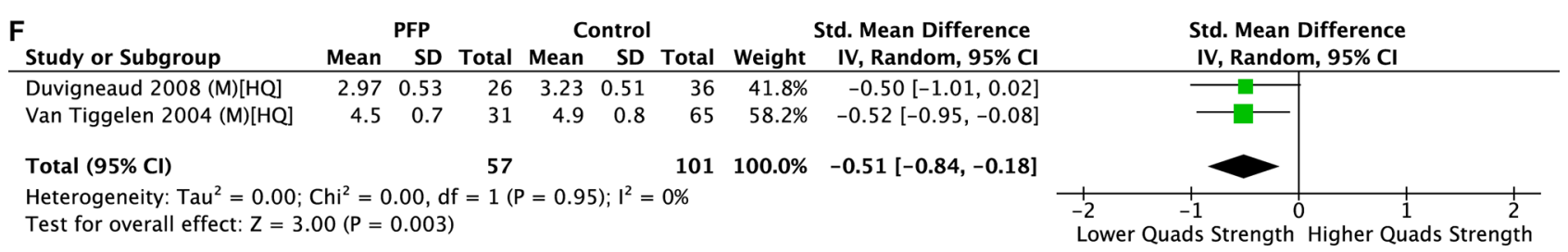

Figure 10 (A-F) Quadriceps strength. Forrest plot detailing standardised mean differences for quadriceps strength when comparing military recruits who developed patellofemoral pain (PFP) with controls. HQ, high quality; IV, inverse variance; M, military recruits. 


\begin{tabular}{|c|c|c|c|c|c|c|c|c|c|}
\hline \multirow{2}{*}{ A } & \multicolumn{3}{|c|}{ PFP } & \multicolumn{3}{|c|}{ Control } & \multicolumn{2}{|c|}{ Std. Mean Difference } & \multirow{2}{*}{$\begin{array}{l}\text { Std. Mean Difference } \\
\text { IV, Random, } 95 \% \mathrm{CI}\end{array}$} \\
\hline & Mean & SD & Total & Mean & SD & Total & Weight & IV, Random, 95\% Cl & \\
\hline Boling $2009(\mathrm{M})[\mathrm{HQ}]$ & 0.3 & 0.07 & 40 & 0.32 & 0.09 & 1279 & $69.1 \%$ & $-0.22[-0.54,0.09]$ & \\
\hline Finnoff $2011(\mathrm{~A})[\mathrm{MQ}]$ & 3.15 & 0.79 & 5 & 2.87 & 0.79 & 92 & $8.4 \%$ & $0.35[-0.55,1.25]$ & \\
\hline Thijs 2011 (R)[MQ] & 0.4 & 0.16 & 16 & 0.43 & 0.12 & 61 & $22.5 \%$ & $-0.23[-0.78,0.32]$ & \\
\hline Total $(95 \% \mathrm{Cl})$ & & & 61 & & & 1432 & $100.0 \%$ & $-0.18[-0.44,0.09]$ & \\
\hline \multicolumn{9}{|c|}{$\begin{array}{l}\text { Heterogeneity: } \mathrm{Tau}^{2}=0.00 ; \mathrm{Chi}^{2}=1.44, \mathrm{df}=2(\mathrm{P}=0.49) ; \mathrm{I}^{2}=0 \% \\
\text { Test for overall effect: } \mathrm{Z}=1.32(\mathrm{P}=0.19)\end{array}$} & th $01 \frac{1}{2}{ }^{0}$ Higher Hip Strength \\
\hline$B_{\text {Study or Subaroup }}$ & & PFP & & & ontrol & & & Std. Mean Difference & Std. Mean Difference \\
\hline Study or Subgroup & Mean & SD & Total & Mean & SD & Total & Weight & IV, Random, 95\% CI & IV, Random, 95\% Cl \\
\hline Boling $2009(\mathrm{M})[\mathrm{HQ}]$ & 0.21 & 0.04 & 40 & 0.22 & 0.04 & 1279 & $60.4 \%$ & $-0.25[-0.56,0.06]$ & \\
\hline Finnoff 2011 (A)[MQ] & 1.88 & 0.68 & 5 & 1.68 & 0.4 & 92 & $11.8 \%$ & $0.48[-0.43,1.38]$ & \\
\hline Thijs 2011 (R)[MQ] & 0.2 & 0.04 & 16 & 0.2 & 0.04 & 61 & $27.8 \%$ & $0.00[-0.55,0.55]$ & \\
\hline \multirow{2}{*}{\multicolumn{9}{|c|}{$\begin{array}{l}\text { Heterogeneity: } \text { Tau }^{2}=0.02 ; \mathrm{Chi}^{2}=2.51, \mathrm{df}=2(\mathrm{P}=0.29) ; \mathrm{I}^{2}=20 \% \\
\text { Test for overall effect: } \mathrm{Z}=0.57(\mathrm{P}=0.57)\end{array}$}} & \\
\hline & & & & & & & & & $\begin{array}{ccccc} & 1 & 1 & 1 & 1 \\
-2 & -1 & 0 & 1 & 2 \\
\text { Lower Hip Strength } & \text { Higher Hip Strength }\end{array}$ \\
\hline C Study or Subgroup & Mean & $\begin{array}{l}\text { PFP } \\
\text { SD }\end{array}$ & Total & $\begin{array}{c}\mathrm{Cr} \\
\text { Mean }\end{array}$ & $\begin{array}{c}\text { ontrol } \\
\text { SD }\end{array}$ & Total & Weight & $\begin{array}{l}\text { Std. Mean Difference } \\
\text { IV, Random, } 95 \% \mathrm{CI}\end{array}$ & $\begin{array}{l}\text { Std. Mean Difference } \\
\text { IV, Random, } 95 \% \mathrm{Cl}\end{array}$ \\
\hline Boling $2009(\mathrm{M})[\mathrm{HQ}]$ & 0.21 & 0.04 & 40 & 0.22 & 0.05 & 1276 & $69.0 \%$ & $-0.20[-0.52,0.11]$ & -十 \\
\hline Finnoff $2011(A)[M Q]$ & 1.34 & 0.26 & 5 & 1.44 & 0.31 & 92 & $8.4 \%$ & $-0.32[-1.22,0.58]$ & \\
\hline Thijs 2011 (R)[MQ] & 0.18 & 0.03 & 16 & 0.18 & 0.03 & 61 & $22.6 \%$ & $0.00[-0.55,0.55]$ & \\
\hline Total $(95 \% \mathrm{Cl})$ & & & 61 & & & 1429 & $100.0 \%$ & $-0.17[-0.43,0.10]$ & \\
\hline $\begin{array}{l}\text { Heterogeneity: } \mathrm{Tau}^{2}= \\
\text { Test for overall effect: }\end{array}$ & $00 ; \mathrm{Cr}$ & $\mathrm{hi}^{2}=$ & $.51, \mathrm{df}$ & 2 & & )$; 1^{2}=$ & & & $\begin{array}{ccccc}-1 & -0.5 & 0 & 0.5 & 1 \\
\text { Lower Hip Strength } & \text { Higher Hip Str }\end{array}$ \\
\hline
\end{tabular}

\begin{tabular}{|c|c|c|c|c|c|c|c|c|c|c|}
\hline \multirow{2}{*}{$D_{\text {Study or Subgroup }}$} & \multicolumn{3}{|c|}{ PFP } & \multicolumn{3}{|c|}{ Control } & \multicolumn{2}{|c|}{ Std. Mean Difference } & \multirow{2}{*}{\multicolumn{2}{|c|}{$\begin{array}{l}\text { Std. Mean Difference } \\
\text { IV, Random, } 95 \% \mathrm{CI}\end{array}$}} \\
\hline & Mean & SD & Total & Mean & SD & Total & Weight & IV, Random, 95\% Cl & & \\
\hline Finnoff $2011(\mathrm{~A})[\mathrm{MQ}]$ & 2.87 & 0.45 & 5 & 2.79 & 0.61 & 92 & $27.4 \%$ & $0.13[-0.77,1.03]$ & & \\
\hline Thijs $2011(\mathrm{R})[\mathrm{MQ}]$ & 0.24 & 0.07 & 16 & 0.26 & 0.06 & 61 & $72.6 \%$ & $-0.32[-0.87,0.23]$ & & \\
\hline Total $(95 \% \mathrm{Cl})$ & & & 21 & & & 153 & $100.0 \%$ & $-0.20[-0.67,0.28]$ & & \\
\hline $\begin{array}{l}\text { Heterogeneity: } \mathrm{Tau}^{2}= \\
\text { Test for overall effect }\end{array}$ & $\begin{array}{l}.00 ; \mathrm{Cl} \\
=0.8\end{array}$ & $\begin{array}{l}\mathrm{hi}^{2}=0 \\
1(\mathrm{P}=\end{array}$ & $\begin{array}{l}.70, d f \\
0.42)\end{array}$ & $=1(P$ & & 0); $1^{2}=$ & & & $\begin{array}{cccc}-2 & -1 & 0 & 1 \\
\text { Lower Hip Strength } & \text { Higher } \mathrm{H}\end{array}$ & $\begin{array}{l}2 \\
\text { Stre }\end{array}$ \\
\hline
\end{tabular}

\begin{tabular}{|c|c|c|c|c|c|c|c|c|c|}
\hline \multirow{2}{*}{$\mathbf{E}_{\text {Study or Subgroup }}$} & \multicolumn{3}{|c|}{ PFP } & \multicolumn{3}{|c|}{ Control } & \multicolumn{2}{|r|}{ Std. Mean Difference } & \multirow{2}{*}{$\begin{array}{l}\text { Std. Mean Difference } \\
\text { IV, Random, } 95 \% \mathrm{CI}\end{array}$} \\
\hline & Mean & SD & Total & Mean & SD & Total & Weight & IV, Random, 95\% Cl & \\
\hline Finnoff $2011(\mathrm{~A})[\mathrm{MQ}]$ & 2.49 & 0.92 & 5 & 2.84 & 0.61 & 92 & $39.0 \%$ & $-0.55[-1.46,0.35]$ & \\
\hline Thijs 2011 (R)[MQ] & 0.42 & 0.08 & 16 & 0.4 & 0.09 & 61 & $61.0 \%$ & $0.22[-0.33,0.78]$ & \\
\hline Total $(95 \% \mathrm{Cl})$ & & & 21 & & & 153 & $100.0 \%$ & $-0.08[-0.82,0.67]$ & \\
\hline \multicolumn{9}{|c|}{$\begin{array}{l}\text { Heterogeneity: } \text { Tau }^{2}=0.16 ; \mathrm{Chi}^{2}=2.08, \mathrm{df}=1(\mathrm{P}=0.15) ; \mathrm{I}^{2}=52 \% \\
\text { Test for overall effect: } \mathrm{Z}=0.21(\mathrm{P}=0.83)\end{array}$} & $\begin{array}{cccc}-2 & -1 & 0 & 1 \\
\text { Hip Strength } & \text { Higher Hip }\end{array}$ \\
\hline
\end{tabular}

Figure 11 (A-E) Hip strength. Forrest plot detailing standardised mean differences for hip strength when comparing participants who developed patellofemoral pain (PFP) with controls. A, adolescents; HQ, high quality; IV, inverse variance; M, military recruits; MQ, medium quality; R, recreational runners.

incidence data have been calculated only from included studies, and the addition of other epidemiology studies that do not fit the inclusion criteria of this review would have affected the values reported. It was also not possible to express incidence data relative to a timeframe given the high heterogeneity observed between included studies.

Some included studies provided data that were not suitable for inclusion in a meta-analysis (ie, no mean/SD or raw counts) and efforts to obtain raw data directly from study authors were unsuccessful. Despite the addition of 11 new studies, the ability to pool data was limited, which is partly attributable to the 116 individual variables investigated across the 18 included studies that could not be pooled. A total of eight studies $2629-32343538$ failed to adhere to the rule of $10^{17}$ (ie, ensuring a minimum of 10 PFP events for each variable of interest), resulting in a high risk of bias and reduced methodological quality.
Given the lack of associations identified by this review (pooled data or otherwise), it is sensible to suggest that perhaps the current body of research is not placing appropriate focus on variables of interest. Altered hip and knee kinematics during running are known to have moderate to strong cross sectional associations with $\mathrm{PFP}^{13}$ yet there remains just one prospective investigation of these variables in women runners only. ${ }^{32}$ There is also an emerging evidence base surrounding the association between psychological variables and PFP, with levels of anxiety, depression, catastrophising and fear of movement reported to be elevated in persons with PFP by a recent systematic review. ${ }^{52}$

The prospective studies included within this review have sought to detect an association between single variables and risk of PFP. The inherent limitation of this approach is the inability to consider interactions between multiple variables. Consequently, 


\begin{tabular}{|c|c|c|c|c|c|c|c|c|c|c|}
\hline A & & PFP & & & ontrol & & & Std. Mean Difference & Std. Mean & fference \\
\hline Study or Subgroup & Mean & SD & Total & Mean & SD & Total & Weight & IV, Random, $95 \% \mathrm{CI}$ & IV, Randor & $95 \% \mathrm{Cl}$ \\
\hline Boling $2009(\mathrm{M})[\mathrm{HQ}]$ & 0.35 & 0.09 & 40 & 0.38 & 0.09 & 1279 & $28.5 \%$ & $-0.33[-0.65,-0.02]$ & 一 & \\
\hline Finnoff $2011(\mathrm{~A})[\mathrm{MQ}]$ & 3.14 & 0.63 & 5 & 2.57 & 0.53 & 92 & $18.7 \%$ & $1.06[0.15,1.97]$ & & \\
\hline Herbst $2015(\mathrm{~A})[\mathrm{HQ}]$ & 0.013 & 0.003 & 38 & 0.011 & 0.003 & 217 & $28.0 \%$ & $0.66[0.32,1.01]$ & & $\longrightarrow$ \\
\hline Thijs 2011 (R)[MQ] & 0.29 & 0.08 & 16 & 0.3 & 0.07 & 61 & $24.8 \%$ & $-0.14[-0.69,0.41]$ & & \\
\hline Total $(95 \% \mathrm{Cl})$ & & & 99 & & & 1649 & $100.0 \%$ & $0.25[-0.38,0.88]$ & & \\
\hline $\begin{array}{l}\text { Heterogeneity: } \mathrm{Tau}^{2} \\
\text { Test for overall effect }\end{array}$ & $\begin{array}{l}0.34 ; \mathrm{Ch} \\
\mathrm{Z}=0.79\end{array}$ & $\begin{array}{l}h i^{2}=22 \\
9(P=0 .\end{array}$ & $\begin{array}{l}.17, \mathrm{df} \\
.43)\end{array}$ & $=3(\mathrm{~F}$ & 0.000 & 1); $\left.\right|^{2}=$ & $86 \%$ & & $\begin{array}{l}-1 \\
\text { er Hip Strength }\end{array}$ & $\begin{array}{ll} & 1 \\
\text { Higher Hip }\end{array}$ \\
\hline
\end{tabular}

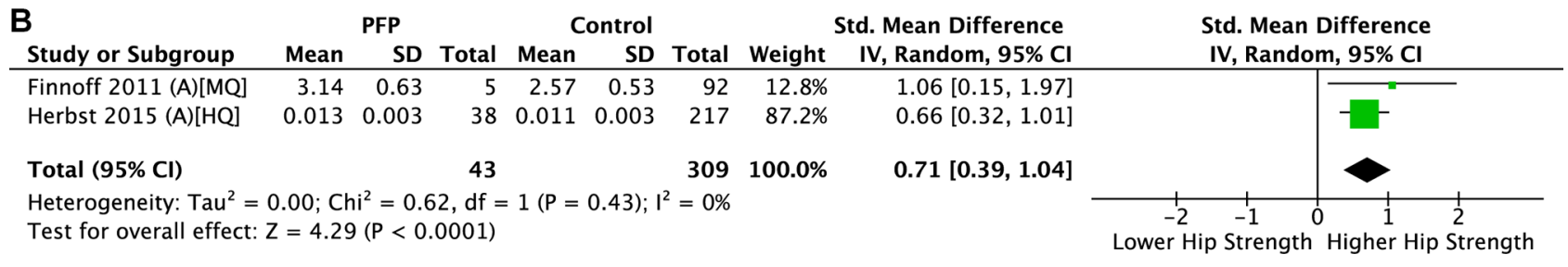

Figure 12 (A, B) Hip abduction strength. Forrest plot detailing standardised mean differences for hip abduction strength when comparing participants who developed patellofemoral pain (PFP) with controls. A, adolescents; HQ, high quality; IV, inverse variance; M, military recruits; MQ, medium quality; $R$, recreational runners.

research needs to move towards a complex systems approach to better understand injury aetiology. ${ }^{53}$ Rather than endeavouring to identify a singular causal factor, studies should be designed to investigate the interactions between a 'web of determinants' that are likely to be non-linear in nature. ${ }^{54}$ This approach has significant methodological challenges and requires the use of a statistical learning approach such as a Bayesian network. ${ }^{55}$ Examples of variables that could fit into a web of determinants for PFP from the published literature include muscle strength (quadriceps and gluteal), hip/knee kinematics, activity levels/sporting workload and psychosocial measures (see figure 13).
No variable included within this systematic review identified a link with future PFP in recreational runners. High peak hip adduction is known to be associated with future PFP in female runners, ${ }^{32}$ and future studies should further explore the causal associations between lower limb kinematics and PFP. While not presented in a fashion that allowed for data pooling, an increased eccentric hip abduction strength reduces the risk of future PFP in recreational runners. ${ }^{33}$ The distinct limitation of this study design is that no guidance was given to the included runners regarding training frequency or intensity, which is likely to be a significant confounder, as more aggressive run volume progressions increase the risk of injury development. ${ }^{56}$

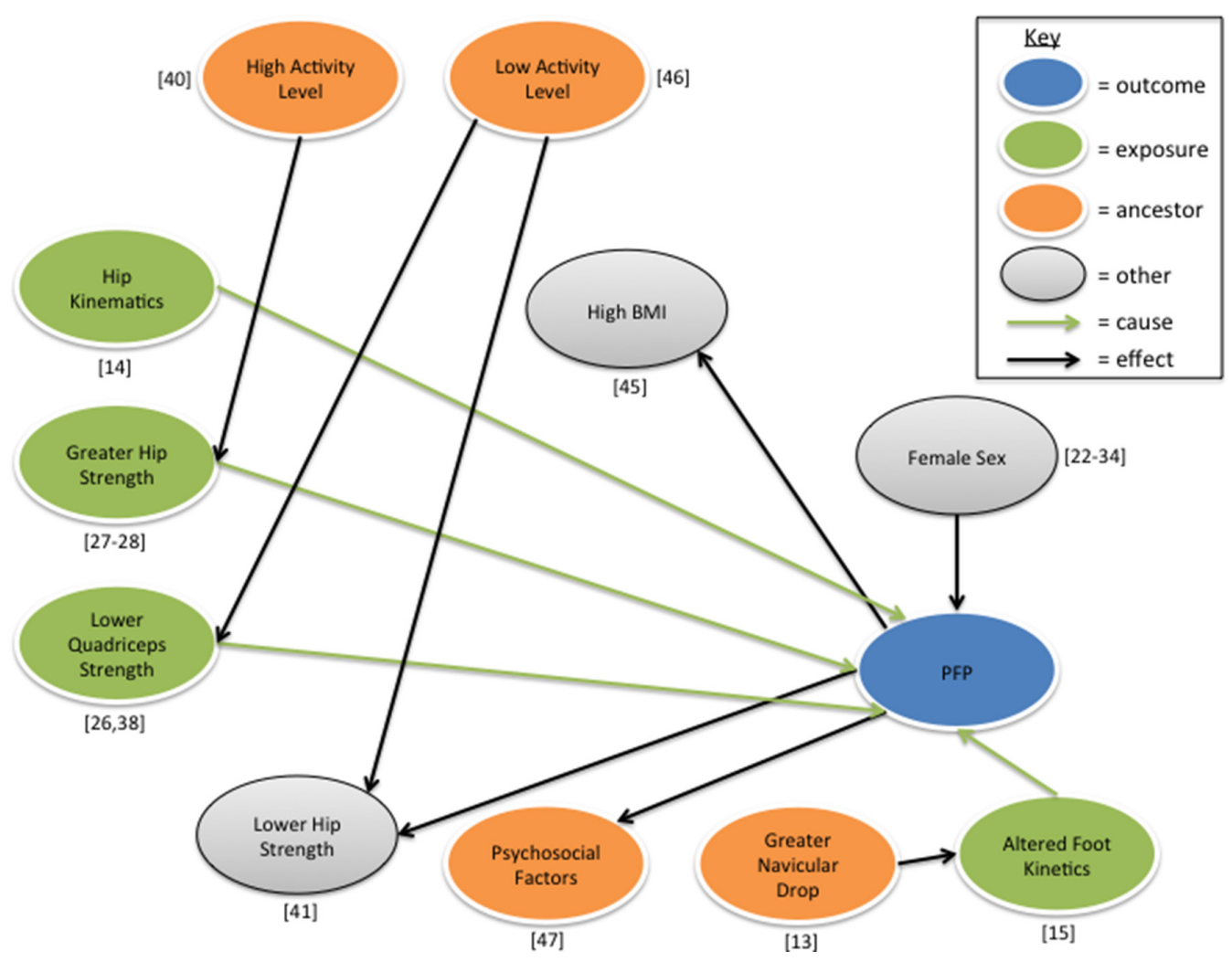

Figure 13 Potential causal inference diagram for patellofemoral pain (PFP). BMI, body mass index. 


\section{CONCLUSION}

Quadriceps weakness, measured using an isokinetic dynamometer and whether or not normalised to either bodyweight or BMI, is a risk factor for future PFP in military recruits and should be investigated as a preventative strategy in a future randomised controlled trial. While increased hip abduction strength is a risk factor for future PFP in adolescents, this may simply be a composite of activity level. Overall, our understanding of what contributes to the development of PFP is inadequate and requires further scientific exploration, although the relationship between given variables and PFP risk is likely to be both complex and individual.

\section{What are the new findings}

Heterogeneous incidence of patellofemoral pain (PFP) is $10 \%$.

- Higher baseline hip abduction strength predicts future PFP development in adolescents.

- Multiple variables (including sex, body mass index and $\mathrm{Q}$ angle) were not found to be risk factors for future PFP development.

Contributors ADR and BSN completed the search. BSN and NEL determined eligible papers for inclusion. SDL and NEL completed the quality appraisal of included papers. BSN and ADR completed the data extraction. BSN, SDL and DHM completed the meta-analysis. All authors contributed to the writing of the final manuscript.

Funding The authors have not declared a specific grant for this research from any funding agency in the public, commercial or not-for-profit sectors.

Competing interests None declared.

Patient consent Not required.

Provenance and peer review Not commissioned; externally peer reviewed.

\section{REFERENCES}

1 Crossley KM, Stefanik JJ, Selfe J, et al. 2016 Patellofemoral pain consensus statement from the 4th International Patellofemoral Pain Research Retreat, Manchester. Part 1: Terminology, definitions, clinical examination, natural history, patellofemoral osteoarthritis and patient-reported outcome measures. Br J Sports Med 2016;50:839-43.

2 Rathleff MS, Skuldbøl SK, Rasch MNB, et al. Care-seeking behaviour of adolescents with knee pain: a population-based study among 504 adolescents. BMC Musculoskelet Disord 2013;14:225.

3 Boling M, Padua D, Marshall S, et al. Gender differences in the incidence and prevalence of patellofemoral pain syndrome. Scand J Med Sci Sports 2010;20:725-30.

4 Smith BE, Selfe J, Thacker D, et al. Incidence and prevalence of patellofemoral pain: A systematic review and meta-analysis. PLoS One 2018;13:e0190892.

5 Callaghan MJ, Selfe J. Has the incidence or prevalence of patellofemoral pain in the general population in the United Kingdom been properly evaluated? Physical Therapy in Sport 2007:8:37-43.

6 Thomas MJ, Wood L, Selfe J, et al. Anterior knee pain in younger adults as a precursor to subsequent patellofemoral osteoarthritis: a systematic review. BMC Musculoskelet Disord 2010;11:201.

7 Crossley KM. Is patellofemoral osteoarthritis a common sequela of patellofemoral pain? Br J Sports Med 2014;48:409-10.

8 Lankhorst NE, van Middelkoop M, Crossley KM, et al. Factors that predict a poor outcome $5-8$ years after the diagnosis of patellofemoral pain: a multicentre observational analysis. Br J Sports Med 2016;50:881-6.

9 van Mechelen W, Hlobil H, Kemper HCG. Incidence, severity, aetiology and prevention of sports injuries. Sports Medicine 1992;14:82-99.

10 Lankhorst NE, Bierma-Zeinstra SM, van Middelkoop M. Risk factors for patellofemoral pain syndrome: a systematic review. J Orthop Sports Phys Ther 2012;42:81-A12.

11 Powers CM, Witvrouw E, Davis IS, et al. Evidence-based framework for a pathomechanical model of patellofemoral pain: 2017 patellofemoral pain consensus statement from the 4th International Patellofemoral Pain Research Retreat, Manchester, UK: part 3. Br J Sports Med 2017;51:1713-23.

12 Neal BS, Griffiths IB, Dowling GJ, et al. Foot posture as a risk factor for lower limb overuse injury: a systematic review and meta-analysis. J Foot Ankle Res 2014;7:55.
13 Neal BS, Barton CJ, Gallie R, et al. Runners with patellofemoral pain have altered biomechanics which targeted interventions can modify: A systematic review and meta-analysis. Gait Posture 2016;45:69-82.

14 Dowling GJ, Murley GS, Munteanu SE, et al. Dynamic foot function as a risk factor for lower limb overuse injury: a systematic review. J Foot Ankle Res 2014;7:53.

15 Liberati A, et al. The PRISMA Statement for Reporting Systematic Reviews and Meta-Analyses of Studies That Evaluate Health Care Interventions: Explanation and Elaboration. Ann Intern Med 2009;151:W-94.

16 Wells G, Shea B, O'connell D, et al, 2008. The Newcastle-Ottawa Scale (NOS) for assessing the quality of nonrandomised studies in meta-analyses. http. ohri. ca/ programs/clinical_epidemiology/oxford. asp (accessed Jun 2014).

17 Peduzzi P, Concato J, Kemper E, et al. A simulation study of the number of events per variable in logistic regression analysis. J Clin Epidemiol 1996;49:1373-9.

18 Hume P, Hopkins W, Rome K, et al. Effectiveness of foot orthoses for treatment and prevention of lower limb injuries: a review. Sports Medicine (Auckland, NZ) 2008;38:759-79.

19 Brockwell SE, Gordon IR. A comparison of statistical methods for meta-analysis. Stat Med 2001;20:825-40.

20 van Tulder M, Furlan A, Bombardier C, et al. Updated method guidelines for systematic reviews in the Cochrane collaboration back review group. Spine 2003:28:1290-9.

21 Boling MC, Padua DA, Marshall SW, et al. A prospective investigation of biomechanical risk factors for patellofemoral pain syndrome: the Joint Undertaking to Monitor and Prevent ACL Injury (JUMP-ACL) cohort. Am J Sports Med 2009;37:2108-16.

22 Barber Foss KD, Hornsby M, Edwards NM, et al. Is body composition associated with an increased risk of developing anterior knee pain in adolescent female athletes? Phys Sportsmed 2012:40:13-19.

23 Milgrom C, Finestone A, Eldad A, et al. Patellofemoral pain caused by overactivity. A prospective study of risk factors in infantry recruits. J Bone Joint Surg Am 1991;73:1041-3.

24 Van Tiggelen D, Cowan S, Coorevits $\mathrm{P}$, et al. Delayed vastus medialis obliquus to vastus lateralis onset timing contributes to the development of patellofemoral pain in previously healthy men: a prospective study. Am J Sports Med 2009:37:1099-105.

25 Duvigneaud N, Bernard E, Stevens V, et al. Isokinetic assessment of patellofemoral pain syndrome: a prospective study in female recruits. Isokinetics and Exercise Science 2008;16:213-9.

26 Finnoff JT, Hall MM, Kyle K, et al. Hip strength and knee pain in high school runners: a prospective study. Pm R 2011;3:792-801.

27 Herbst KA, Barber Foss KD, Fader $L$, et al. Hip strength is greater in athletes who subsequently develop patellofemoral pain. Am J Sports Med 2015;43:2747-52

28 Hetsroni I, Finestone A, Milgrom C, et al. A prospective biomechanical study of the association between foot pronation and the incidence of anterior knee pain among military recruits. J Bone Joint Surg Br 2006;88-B:905-8.

29 Holden S, Boreham C, Doherty C, et al. Two-dimensional knee valgus displacement as a predictor of patellofemoral pain in adolescent females. Scand J Med Sci Sports 2017:27 188- 194.

30 Luedke LE, Heiderscheit BC, Williams DSB, et al. Influence of step rate on shin injury and anterior knee pain in high school Runners. Medicine \& Science in Sports \& Exercise 2016;48:1244-50.

31 Myer GD, Ford KR, Barber Foss KD, et al. The incidence and potential pathomechanics of patellofemoral pain in female athletes. Clin Biomech 2010;25:700-7.

32 Noehren B, Hamill J, Davis I. Prospective evidence for a hip etiology in patellofemoral pain. Med Sci Sports Exerc 2013;45:1120-4

33 Ramskov D, Barton C, Nielsen RO, et al. High eccentric hip abduction strength reduces the risk of developing patellofemoral pain among novice runners initiating a selfstructured running program: a 1-year observational study. J Orthop Sports Phys Ther 2015:45:153-61.

34 Thijs Y, De Clercq D, Roosen $\mathrm{P}$, et al. Gait-related intrinsic risk factors for patellofemoral pain in novice recreational runners. Br J Sports Med 2008;42:466-71.

35 Thijs Y, Pattyn E, Van Tiggelen D, et al. Is hip muscle weakness a predisposing factor for patellofemoral pain in female novice runners? A prospective study. Am J Sports Med 2011:39:1877-82

36 Thijs Y, Van Tiggelen D, Roosen $P$, et al. A prospective study on gait-related intrinsic risk factors for patellofemoral pain. Clin J Sport Med 2007;17:437-45.

37 Van Tiggelen D, Witvrouw E, Coorevits $\mathrm{P}$, et al. Analysis of isokinetic parameters in the development of anterior knee pain syndrome: a prospective study in a military setting. Isokinetics and Exercise Science 2004;12:223-8.

38 Witvrouw E, Lysens R, Bellemans J, et al. Intrinsic risk factors for the development of anterior knee pain in an athletic population a two-year prospective study. Am J Sports Med 2000;28:480-9.

39 Rathleff CR, Baird WN, Olesen JL, et al. Hip and knee strength is not affected in 12-16 year old adolescents with patellofemoral pain-a cross -sectional population-based study. PloS One 2013;8:e79153.

40 Rathleff MS, Rathleff CR, Crossley KM, et al. Is hip strength a risk factor for patellofemoral pain? A systematic review and meta-analysis. Br J Sports Med 2014;48:1088 
41 Lankhorst NE, Bierma-Zeinstra SMA, van Middelkoop M. Factors associated with patellofemoral pain syndrome: a systematic review. Br J Sports Med 2013;47:193-206.

42 Fukuda TY, Melo WP, Zaffalon BM, et al. Hip posterolateral musculature strengthening in sedentary women with patellofemoral pain syndrome: a randomized controlled clinical trial with 1-year follow-up. J Orthop Sports Phys Ther 2012;42:823-30.

43 van Linschoten R, van Middelkoop M, Berger MY, et al. Supervised exercise therapy versus usual care for patellofemoral pain syndrome: an open label randomised controlled trial. BMJ (Clinical Research Edn) 2009;339:b4074.

44 Rathleff MS, Roos EM, Olesen JL, et al. Exercise during school hours when added to patient education improves outcome for 2 years in adolescent patellofemoral pain: a cluster randomised trial. Br J Sports Med 2015;49:406-12.

45 Hannigan JJ, Osternig LR, Chou LS. Sex-Specific Relationships between hip strength and hip, pelvis, and trunk kinematics in healthy runners. J App/ Biomech 2018;34:1-22.

46 Rathleff MS, Rathleff CR, Olesen JL, et al. Is knee pain during adolescence a selflimiting condition? Prognosis of patellofemoral pain and other types of knee pain. Am J Sports Med 2016:44:1165-71.

47 Coppack RJ, Etherington J, Wills AK. The effects of exercise for the prevention of overuse anterior knee pain: a randomized controlled trial. Am J Sports Med 2011;39:940-8.
48 Hart HF, Barton CJ, Khan KM, et al. Is body mass index associated with patellofemoral pain and patellofemoral osteoarthritis? A systematic review and meta-regression and analysis. Br J Sports Med 2017;51:781-90.

49 Glaviano NR, Baellow A, Saliba S. Physical activity levels in individuals with and without patellofemoral pain. Physical Therapy in Sport 2017;27:12-16.

50 Wills AK, Ramasamy A, Ewins DJ, et al. The incidence and occupational outcome of overuse anterior knee pain during army recruit training. J R Army Med Corps 2004;150:264-9.

51 Kaufman KR, Brodine SK, Shaffer RA, et al. The effect of foot structure and range of motion on musculoskeletal overuse injuries. Am J Sports Med 1999;27:585-93.

52 Maclachlan LR, Collins NJ, Matthews MLG, et al. The psychological features of patellofemoral pain: a systematic review. Br J Sports Med 2017;51:732-42.

53 Bittencourt NFN, Meeuwisse WH, Mendonça LD, et al. Complex systems approach for sports injuries: moving from risk factor identification to injury pattern recognitionnarrative review and new concept. Br J Sports Med 2016:50:1309-14.

54 Philippe $\mathrm{P}$, Mansi $\mathrm{O}$. Nonlinearity in the epidemiology of complex health and disease processes. Theor Med Bioeth 1998;19:591-607.

55 Chipman HA, George El, McCulloch RE. BART: Bayesian additive regression trees. Ann App/ Stat 2010;4:266-98

56 Nielsen RØ, Parner ET, Nohr EA, et al. Excessive progression in weekly running distance and risk of running-related injuries: an association which varies according to type of injury. J Orthop Sports Phys Ther 2014;44:739-47. 